\title{
Angular analysis of two classes of non-polyhedral convex cones: the point of view of optimization theory*
}

\author{
ALFREDO IUSEM $^{1}$ and ALBERTO SEEGER ${ }^{2}$ \\ ${ }^{1}$ Instituto de Matemática Pura e Aplicada, Estrada Dona Castorina 110 \\ Jardim Botânico, Rio de Janeiro, Brazil \\ ${ }^{2}$ University of Avignon, Department of Mathematics, 33 rue Pasteur, 84000 Avignon, France \\ E-mails: iusp@impa.br / alberto.seeger@univ-avignon.fr
}

\begin{abstract}
There are three related concepts that arise in connection with the angular analysis of a convex cone: antipodality, criticality, and Nash equilibria. These concepts are geometric in nature but they can also be approached from the perspective of optimization theory. A detailed angular analysis of polyhedral convex cones has been carried out in a recent work of ours. This note focus on two important classes of non-polyhedral convex cones: elliptic cones in an Euclidean vector space and spectral cones in a space of symmetric matrices.
\end{abstract}

Mathematical subject classification: 52A40, 90C26.

Key words: antipodal pairs, convex cones, maximal angle, critical angle, Nash angular equilibria, elliptic cones, spectral cones.

\section{Introduction}

The Euclidean space $\mathbb{R}^{d}$ is equipped with the usual inner product $\langle u, v\rangle=u^{T} v$ and the associated norm $\|\cdot\|$. The symbol $\Sigma_{d}$ refers to the unit sphere in $\mathbb{R}^{d}$. We also use the notation

$$
\Xi\left(\mathbb{R}^{d}\right) \equiv \text { nontrivial closed convex cones in } \mathbb{R}^{d} .
$$

\#703/07. Received: 08/II/07. Accepted: 07/III/07.

* Research carried out within the framework of the Brazil-France Cooperation Agreement in Mathematics. 
That a convex cone $K$ in $\mathbb{R}^{d}$ is nontrivial means that $K$ is different from $\{0\}$ and different from the space $\mathbb{R}^{d}$ itself. The symbol $K^{+}$is used to indicate the positive dual cone of $K$.

There are various tools that serve to describe the angular structure of a convex cone. The following definition recalls the main conceptual ingredients used in this note.

Definition 1. Let $K \in \Xi\left(\mathbb{R}^{d}\right)$. Let $\bar{u}$ and $\bar{v}$ be two unit vectors in $K$.

i) $(\bar{u}, \bar{v})$ is an antipodal pair of $K$ if $\bar{u}$ and $\bar{v}$ achieve the maximal angle

$$
\theta_{\max }(K)=\sup _{u, v \in K \cap \Sigma_{d}} \arccos \langle u, v\rangle .
$$

ii) The angle $\theta(\bar{u}, \bar{v})=\arccos \langle\bar{u}, \bar{v}\rangle$ formed by a critical pair $(\bar{u}, \bar{v})$ is called a critical angle. That a pair $(\bar{u}, \bar{v})$ is critical means that

$$
\bar{v}-\langle\bar{u}, \bar{v}\rangle \bar{u} \in K^{+} \quad \text { and } \quad \bar{u}-\langle\bar{u}, \bar{v}\rangle \bar{v} \in K^{+} .
$$

The adjective proper is added when $\bar{u}$ and $\bar{v}$ are not collinear, i.e., $|\langle\bar{u}, \bar{v}\rangle|$ $\neq 1$. The set of all proper critical angles of $K$, denoted by $\Omega(K)$, is called the angular spectrum of $K$.

iii) $(\bar{u}, \bar{v})$ is a Nash angular equilibrium of $K$ if

$$
\begin{array}{cl}
\theta(\bar{u}, \bar{v}) \geq \theta(\bar{u}, v) & \forall v \in K \cap \Sigma_{d}, \\
\theta(\bar{u}, \bar{v}) \geq \theta(u, \bar{v}) & \forall u \in K \cap \Sigma_{d} .
\end{array}
$$

The number $\theta(\bar{u}, \bar{v})$ is then called a Nash angle of $K$.

The motivation behind each of the above concepts is explained with great care and detail in our previous work [8]. For the reader's convenience, we recall some of the reasons why the study of critical angles is important. The largest critical angle of a convex cone $K$ is $\theta_{\max }(K)$, i.e., the maximal angle that can be formed by picking up two unit vectors from $K$. The geometric meaning of $\theta_{\max }(K)$ justifies by itself the study of the variational problem (1), but there are also application-oriented motivations. For instance, Peña and Renegar [13] 
show that the number $\theta_{\max }(K)$ plays a role in estimating the efficiency of certain interior point methods for solving feasibility systems with inequalities described by $K$. On the other hand, $\theta_{\max }(K)$ is related to

$$
\rho(K)=\min _{\substack{Q \in \Xi\left(\mathbb{R}^{d}\right) \\ Q \text { unpointed }}} \operatorname{haus}(K, Q),
$$

a number which has been suggested in [4] as tool for measuring the degree of pointedness of $K$. Here

$$
\operatorname{haus}(K, Q)=\max \left\{\max _{x \in K \cap \Sigma_{d}} \operatorname{dist}(x, Q), \max _{x \in Q \cap \Sigma_{d}} \operatorname{dist}(x, K)\right\}
$$

stands for the bounded Pompeiu-Hausdorff metric on $\Xi\left(\mathbb{R}^{d}\right)$. In general, the evaluation of (2) is a cumbersome task even for cones having a relatively simple structure. Fortunately, the least distance problem (2) is related to the angle maximization problem (1) which, in principle, is easier to solve because the decision variables $u, v$ live in a standard Euclidean space. In fact, one has the following striking formula [9]

$$
\rho(K)=\cos \left[\frac{\theta_{\max }(K)}{2}\right] .
$$

Moreover, if $K$ is not a half-line and admits $(\bar{u}, \bar{v})$ as antipodal pair, then the closed convex cone

$$
Q=K \cap[\mathbb{R}(\bar{u}-\bar{v})]^{\perp}+\mathbb{R}(\bar{u}-\bar{v})
$$

is unpointed and lies at minimal distance from $K$.

Of course, $\theta_{\max }(K)$ is not the only critical angle of interest. The smallest proper critical angle plays also a relevant role in the description of the cone, namely, it can be used as tool for measuring its degree of solidity. By an index of solidity we understand any continuous function $G: \Xi\left(\mathbb{R}^{d}\right) \rightarrow \mathbb{R}$ satisfying the axioms:

i) $G(K)=0$ if and only if $K$ is not solid,

ii) $G(K)=1$ if and only if $K$ is a half-space,

iii) $G(U(K))=G(K)$ for any orthonormal matrix $U$,

iv) $K_{1} \subset K_{2}$ implies $G\left(K_{1}\right) \leq G\left(K_{2}\right)$. 
The Frobenius coefficient

$$
G_{\text {frob }}(K)=\left\{\begin{array}{l}
\text { radius of the largest ball contained } \\
\text { in } K \text { and centered in a unit vector }
\end{array}\right.
$$

is the first example of index of solidity that comes to mind. An alternative choice is

$$
G_{\text {angular }}(K)=\rho\left(K^{+}\right)=\cos \left[\frac{\theta_{\max }\left(K^{+}\right)}{2}\right] .
$$

What is bothering about the expression (3) is that it involves the dual cone $K^{+}$ and not the original cone $K$ itself. However, this problem can be remediated since it is possible to write (3) in the equivalent form

$$
G_{\text {angular }}(K)=\left\{\begin{array}{cl}
\sin \left[\frac{\theta_{\min }(K)}{2}\right] & \text { if } K \text { is solid } \\
0 & \text { if } K \text { is not solid, }
\end{array}\right.
$$

where $\theta_{\min }(K)$ indicates the smallest proper critical angle of $K$.

We have explained in few words why the maximal angle and the smallest proper critical angle are mathematical objects of interest. The intermediate critical angles are perhaps less useful, but in any case they provide additional information on the geometric structure of the cone. We now come back to the main stream of the presentation. The main fact to be remembered about Definition 1 is that every antipodal pair is a Nash angular equilibrium, and that every Nash angular equilibrium is a critical pair.

It is useful to split the angular spectrum of $K$ in two disjoint pieces:

$$
\Omega(K)=\Omega_{\text {nash }}(K) \cup \Omega_{\text {ord }}(K) .
$$

The first piece collects the proper critical angles that are formed by a Nash angular equilibrium. The remaining proper critical angles are said to be "ordinary" and they are thrown in the set $\Omega_{\text {ord }}(K)$.

The angular structure of a polyhedral convex cone is by now well understood as one can see by consulting the references $[5,7,8]$. In a non-polyhedral setting the situation is more involved as one may expect. For instance, in the technical note [6] we succeeded in constructing a non-polyhedral convex cone whose angular spectrum is uncountable, a phenomenon that cannot occur under polyhedrality. The example constructed in [6] is very involved and its interest is mainly academic. 
In this note we study two important classes of non-polyhedral convex cones arising in practice: elliptic cones in an Euclidean vector space and spectral cones in a space of symmetric matrices.

\section{Angular Analysis of Elliptic Cones}

In this section we consider $d=n+1$ with $n \geq 2$. Recall that the elliptic cone $\mathcal{E}(A)$ associated to a symmetric positive definite matrix $A \in \mathbb{R}^{n \times n}$ is the closed convex cone in $\mathbb{R}^{n+1}$ given by

$$
\mathcal{E}(A)=\left\{(x, r) \in \mathbb{R}^{n+1}: \sqrt{x^{T} A x} \leq r\right\} .
$$

Elliptic cones are used in a great diversity of areas. The three dimensional case has applications in contact problems with orthotropic friction law [3, 17] and in electromagnetic scattering [16], just to mention two concrete examples. General background on higher dimensional elliptic cones can be found in [4, 7] among other references.

The trace of the elliptic cone $\mathcal{E}(A)$ over the unit sphere $\Sigma_{n+1}$ is the set all vectors $(x, r) \in \mathbb{R}^{n+1}$ such that

$$
\begin{aligned}
\sqrt{x^{T} A x} & \leq r, \\
\|x\|^{2}+r^{2} & =1 .
\end{aligned}
$$

The Cartesian representation (4)-(5) is not always the best way of describing $\mathcal{E}(A) \cap \Sigma_{n+1}$. As explained in the next lemma, this set can also be described by using a parametric representation. The notation

$$
e(C)=\left\{\alpha \in \mathbb{R}^{n}:\langle\alpha, C \alpha\rangle \leq 1\right\}
$$

stands for the ellipsoid associated to a symmetric positive definite matrix $C \in$ $\mathbb{R}^{n \times n}$.

Lemma 1. Decompose the symmetric positive definite matrix $A \in \mathbb{R}^{n \times n}$ in the usual spectral form $A=Q D Q^{T}$, where $D$ is a diagonal matrix containing the eigenvalues of $A$ arranged in nondecreasing order $\mu_{1} \leq \cdots \leq \mu_{n}$, 
and $Q=\left[q_{1} \cdots q_{n}\right]$ is an orthonormal matrix whose columns are formed with corresponding eigenvectors $q_{1}, \ldots, q_{n}$. Then,

$$
z \in \mathcal{E}(A) \cap \Sigma_{n+1} \Longleftrightarrow z=\left(Q \alpha, \sqrt{1-\|\alpha\|^{2}}\right) \text { with } \quad \alpha \in e(I+D) .
$$

Proof. Take any $z=(x, r)$ and write its first component in the form $x=$ $Q \alpha$ with $\alpha \in \mathbb{R}^{n}$. The fact that $z$ has unit length is expressed by the relation $r=\sqrt{1-\|\alpha\|^{2}}$. Membership of $z$ in $\mathcal{E}(A)$ corresponds to the inequality $\langle\alpha,(I+D) \alpha\rangle \leq 1$.

We refer to (6) as the canonical parametrization of $\mathcal{E}(A) \cap \Sigma_{n+1}$. Since $e(I+D)$ is contained in $B_{n}$, the closed unit ball of $\mathbb{R}^{n}$, the square root operation in (6) is well defined. For the sake of convenience, we introduce the function $\Psi: B_{n} \rightarrow \mathbb{R}^{n+1}$ given by

$$
\Psi(\alpha)=\left(Q \alpha, \sqrt{1-\|\alpha\|^{2}}\right) .
$$

Although $\Psi$ depends explicitly on the collection $\left\{q_{1}, \ldots q_{n}\right\}$ of eigenvectors of $A$, the spherical product

$$
(\alpha, \beta) \in B_{n} \times B_{n} \mapsto \Phi(\alpha, \beta)=\langle\Psi(\alpha), \Psi(\beta)\rangle
$$

is a more intrinsic concept. Indeed, by orthonormality of $Q$, one simply has

$$
\Phi(\alpha, \beta)=\langle\alpha, \beta\rangle+\sqrt{1-\|\alpha\|^{2}} \sqrt{1-\|\beta\|^{2}} .
$$

There is a very interesting theory behind the definition of $\Phi$, but we shall not elaborate on this subject more than strictly necessary. The use of this special type of vector product will be clear in a moment.

\subsection{Antipodal and critical pairs in $\mathcal{E}(A)$}

In what follows we use the parametrization of $\mathcal{E}(A) \cap \Sigma_{n+1}$ described in Lemma 1. Arbitrary points in $\mathcal{E}(A) \cap \Sigma_{n+1}$, say $u$ and $v$, will be represented in the parametric form

$$
u=\Psi(\alpha), \quad v=\Psi(\beta), \quad \text { with } \quad \alpha, \beta \in e(I+D) .
$$


Since $\Psi$ is a bijection between $e(I+D)$ and $\mathcal{E}(A) \cap \Sigma_{n+1}$, the angle maximization problem (1) becomes

$$
\begin{aligned}
& \text { minimize }\langle\alpha, \beta\rangle+\sqrt{1-\|\alpha\|^{2}} \sqrt{1-\|\beta\|^{2}} \\
& \text { subject to } \alpha, \beta \in e(I+D) .
\end{aligned}
$$

A careful analysis of the above variational problem leads to a full characterization of the set of antipodal pairs of $\mathcal{E}(A)$.

Theorem 1. Decompose the symmetric positive definite matrix $A \in \mathbb{R}^{n \times n}$ as in Lemma 1. Suppose that the smallest eigenvalue of $A$, denoted by $\mu_{\min }(A)$, has multiplicity $r$, that is to say, the eigenspace associated to $\mu_{\min }(A)$ is $r$ dimensional. Then,

(a) the maximal angle of $\mathcal{E}(A)$ is given by

$$
\theta_{\max }(\mathcal{E}(A))=\arccos \left[\frac{\mu_{\min }(A)-1}{\mu_{\min }(A)+1}\right] .
$$

(b) $(\bar{u}, \bar{v})$ is an antipodal pair of $\mathcal{E}(A)$ if and only

$$
\bar{u}=\left(\sum_{i=1}^{r} \alpha_{i} q_{i}, \sqrt{\frac{\mu_{\min }(A)}{\mu_{\min }(A)+1}}\right), \quad \bar{v}=\left(-\sum_{i=1}^{r} \alpha_{i} q_{i}, \sqrt{\frac{\mu_{\min }(A)}{\mu_{\min }(A)+1}}\right),
$$

with coefficients $\alpha_{1}, \ldots, \alpha_{r} \in \mathbb{R}$ such that

$$
\sum_{i=1}^{r} \alpha_{i}^{2}=\frac{1}{\mu_{\min }(A)+1}
$$

Proof. Given the specific structure of (7), one sees that this minimization problem is solved by taking $\alpha_{r+1}=\cdots=\alpha_{n}=0$ and the first $r$ coefficients of $\alpha$ as in (8). The parameter vector $\beta$ must have opposite orientation with respect to $\alpha$, i.e. we must take $\beta=-\alpha$. Part (a) follows immediately from (b). 
Remark 1. If the eigenvalue $\mu_{\min }(A)$ is simple, that is to say, $r=1$, then the elliptic cone $\mathcal{E}(A)$ has exactly two antipodal pairs, namely,

$$
\begin{aligned}
& \bar{u}=\frac{1}{\sqrt{\mu_{\min }(A)+1}}\left( \pm q_{1}, \sqrt{\mu_{\min }(A)}\right), \\
& \bar{v}=\frac{1}{\sqrt{\mu_{\min }(A)+1}}\left(\mp q_{1}, \sqrt{\mu_{\min }(A)}\right) .
\end{aligned}
$$

Since one antipodal pair is obtained from the other by permuting the order of $u$ and $v$, one may say that the antipodal pair is "unique". In case of higher order multiplicity, i.e. $r \geq 2$, the collection of antipodal pairs can be parametrized with an $r$-dimensional parameter vector as in (8).

We recall a result on angular spectra of elliptic cones obtained recently in [7]. As shown in the next theorem, computing the angular spectrum of $\mathcal{E}(A)$ is essentially the same job as computing all the eigenvalues of the matrix $A$. The critical pairs of $\mathcal{E}(A)$ are obtained by using the eigenvectors of $A$.

Theorem 2. Let $A \in \mathbb{R}^{n \times n}$ be symmetric and positive definite. The vectors $(x, r)$ and $(y, s)$ form a proper critical pair of $\mathcal{E}(A)$ if and only if the following three conditions hold:

(a) $y=-x$,

(b) $s=r=\sqrt{1-\|x\|^{2}}=\sqrt{x^{T} A x}$,

(c) $x$ is an eigenvector of $A$.

In this case, the corresponding critical angle takes the value

$$
\theta=\arccos \left(\frac{\mu-1}{\mu+1}\right)
$$

where $\mu$ is the eigenvalue of $A$ associated to $x$.

The proof technique used in [7] doesn't rely on the canonical parametrization of $\mathcal{E}(A) \cap \Sigma_{n+1}$. By relying on Lemma 1 it is possible to describe in a more precise manner the nature of a given critical pair of $\mathcal{E}(A)$ in terms of the multiplicity of the corresponding eigenvalue of $A$. We will not indulge however on this matter. Theorem 2 as stated is all what we need to go on with our exposition. 


\subsection{Nash angular equilibria in $\mathcal{E}(A)$}

Elliptic cones are, no doubt, very special objects. According to Theorem 2, the angular spectrum of $\mathcal{E}(A)$ has at most $n$ elements. Indeed,

$$
\Omega(\mathcal{E}(A))=\left\{\theta_{1}, \ldots, \theta_{n}\right\},
$$

where the $i$-th critical angle

$$
\theta_{i}=\arccos \left(\frac{\mu_{i}-1}{\mu_{i}+1}\right)
$$

is obtained directly from the $i$-th eigenvalue $\mu_{i}$ of $A$.

The theorem stated below is a fundamental result on Nash angular equilibria of elliptic cones. Several consequences of this theorem will be presented as soon as the proof is completed.

Theorem 3. Let $(\bar{u}, \bar{v})$ be a proper critical pair of $\mathcal{E}(A)$. Denote by $\theta$ the corresponding critical angle and by $\mu$ the corresponding eigenvalue, that is to say, $\mu$ is the unique solution of the equation (9). The following four conditions are then equivalent:

(a) $(\bar{u}, \bar{v})$ is a Nash angular equilibrium of $\mathcal{E}(A)$,

(b) $\bar{u}$ minimizes the linear form $\langle\cdot, \bar{v}\rangle$ over $\mathcal{E}(A) \cap \Sigma_{n+1}$,

(c) $\bar{v}$ minimizes the linear form $\langle\bar{u}, \cdot\rangle$ over $\mathcal{E}(A) \cap \Sigma_{n+1}$,

(d) $\mu \leq 1+2 \mu_{\min }(A)$

Proof. Take, for instance, $\theta=\theta_{k}$, that is to say, consider the critical angle that derives from $\mu=\mu_{k}$. As in Lemma 1 , we form $Q=\left[q_{1} \cdots q_{n}\right]$ with an orthonormal basis of eigenvectors of $A$. The diagonal matrix $D$ is formed with the corresponding eigenvalues that we arrange in nondecreasing order $\mu_{\min }(A)=$ $\mu_{1} \leq \cdots \leq \mu_{n}$. Either by using the parametric representation of $\bar{u}$ and $\bar{v}$ as in Lemma 1 , or by applying Theorem 2, one gets

$$
\begin{aligned}
& \bar{u}=\frac{1}{\sqrt{1+\mu}}\left(q_{k}, \sqrt{\mu}\right), \\
& \bar{v}=\frac{1}{\sqrt{1+\mu}}\left(-q_{k}, \sqrt{\mu}\right) .
\end{aligned}
$$


For the sake of clarity, we divide the proof of the theorem in five steps.

Step 1: We study the behavior of $\langle\bar{u}, \cdot\rangle$ over a special path. For each $t \in[0,1]$, consider the point

$$
z_{t}=\left(1+r_{t}^{2}\right)^{-1 / 2}\left(x_{t}, r_{t}\right)
$$

with

$$
x_{t}=-\sqrt{t} q_{k}+\sqrt{1-t} q_{1}, \quad \text { and } \quad r_{t}=\sqrt{x_{t}^{T} A x_{t}} .
$$

Note that $x_{t}$ has unit length, and so does $z_{t}$. Note also that, in view of the equality in the definition of $r_{t}$, the vector $z_{t}$ belongs to bd[ $\left.\mathcal{E}(A)\right]$, the boundary of $\mathcal{E}(A)$. In short, $\left\{z_{t}: t \in[0,1]\right\}$ is a continuous path on the set $\Sigma_{n+1} \cap \operatorname{bd}[\mathcal{E}(A)]$. We will study the behavior of the univariate function $\phi:[0,1] \rightarrow \mathbb{R}$ given by $\phi(t)=\left\langle\bar{u}, z_{t}\right\rangle$. In view of (10) and the definition of $z_{t}$, after some simplification one arrives at the expression

$$
\phi(t)=\frac{-\sqrt{t}+\sqrt{\mu} \sqrt{t \mu+(1-t) \mu_{1}}}{\sqrt{1+\mu} \sqrt{1+t \mu+(1-t) \mu_{1}}} .
$$

Observe that $\phi(1)=(\mu-1) /(\mu+1)=\langle\bar{u}, \bar{v}\rangle$. Let us examine the sign of $\phi^{\prime}(1)$. Since

$$
\phi^{\prime}(t)=\frac{\left[\frac{-1}{\sqrt{t}}+\frac{\sqrt{\mu}\left(\mu_{1}-\mu_{1}\right)}{\sqrt{t\left(\mu-\mu_{1}\right)+\mu_{1}}}\right] \sqrt{t\left(\mu-\mu_{1}\right)+\mu_{1}+1}-\frac{\left(-\sqrt{t}+\sqrt{\mu} \sqrt{t\left(\mu-\mu_{1}\right)+\mu_{1}}\right)\left(\mu-\mu_{1}\right)}{\sqrt{t\left(\mu-\mu_{1}\right)+\mu_{1}+1}}}{2\left[t\left(\mu-\mu_{1}\right)+\mu_{1}+1\right] \sqrt{\mu+1}},
$$

one gets

$$
\begin{aligned}
\phi^{\prime}(1) & =\frac{1}{2(\mu+1)^{3 / 2}}\left[\left(-1+\frac{\sqrt{\mu}\left(\mu-\mu_{1}\right)}{\sqrt{\mu}}\right) \sqrt{\mu+1}-\frac{(-1+\sqrt{\mu} \sqrt{\mu})\left(\mu-\mu_{1}\right)}{\sqrt{\mu+1}}\right] \\
& =\frac{1}{2(\mu+1)^{2}}\left(\mu-2 \mu_{1}-1\right) .
\end{aligned}
$$

With this information at hand, we can proceed with the next step.

Step 2: We prove that $(c) \Rightarrow(d)$. Suppose on the contrary that $\mu>2 \mu_{1}-1$. So $\phi^{\prime}(1)>0$, and therefore

$$
\left\langle\bar{u}, z_{t}\right\rangle=\phi(t)<\phi(1)=\langle\bar{u}, \bar{v}\rangle
$$


for some $t \in[0,1]$ closed enough to 1 . Since $z_{t}$ belongs to $\mathcal{E}(A) \cap \Sigma_{n+1}$ for all $t \in[0,1]$, the vector $\bar{v}$ is not a minimizer of $\langle\bar{u}, \cdot\rangle$ over $\mathcal{E}(A) \cap \Sigma_{n+1}$. This contradiction confirms that (c) implies (d).

Step 3: We derive a so-called "extrapolation property" associated to condition (c). Consider the inequality

$$
\langle\bar{u}, \bar{v}\rangle \leq\langle\bar{u}, v\rangle \quad \forall v \in \mathcal{E}(A) \cap \Sigma_{n+1} .
$$

We represent a point $v \in \mathcal{E}(A) \cap \Sigma_{n+1}$ in terms of a parameter vector $\beta \in \mathbb{R}^{n}$ as described in Lemma 1, i.e.

$$
v=\Phi(\beta) \quad \text { with } \quad \beta \in e(I+D)
$$

It follows that, for $v$ as in (12), one has

$$
\langle\bar{u}, v\rangle=\frac{1}{\sqrt{1+\mu}}\left(\beta_{k}+\sqrt{\mu} \sqrt{1-\|\beta\|^{2}}\right) .
$$

For convenience we make the change of variables

$$
r=\frac{\sqrt{1-\|\beta\|^{2}}}{\|\beta\|}, \quad \delta_{i}=\frac{\beta_{i}}{\|\beta\|} \quad \text { for } \quad i=1, \ldots, n .
$$

Thus,

$$
\begin{aligned}
\langle\bar{u}, v\rangle & =\frac{\delta_{k}+r \sqrt{\mu}}{\sqrt{(1+\mu)\left(1+r^{2}\right)}} \geq \frac{-\left|\delta_{k}\right|+r \sqrt{\mu}}{\sqrt{(1+\mu)\left(1+r^{2}\right)}} \\
& =\frac{-\left|\delta_{k}\right|}{\sqrt{(1+\mu)\left(1+r^{2}\right)}}+\frac{\sqrt{\mu}}{\sqrt{(1+\mu)\left(1+\frac{1}{r^{2}}\right)}} .
\end{aligned}
$$

The rightmost expression in (13) is clearly an increasing function of $r$ on the positive half-line. Observe that, since $v$ belongs to $\mathcal{E}(A)$ and $\mu_{i} \geq \mu_{1}(1 \leq i \leq$ $n$ ), it holds that

$$
\begin{aligned}
r^{2} \geq \sum_{i=1}^{n} \delta_{i}^{2} \mu_{i} & =\delta_{k}^{2} \mu+\sum_{i \neq k} \delta_{i}^{2} \mu_{i} \geq \delta_{k}^{2} \mu+\left(\sum_{i \neq k} \delta_{i}^{2}\right) \mu_{1} \\
& =\delta_{k}^{2} \mu+\left(1-\delta_{k}^{2}\right) \mu_{1},
\end{aligned}
$$


using the facts that $\sum_{i=1}^{n} \delta_{i}^{2}=1$ in the rightmost equality of (14). Replacing (14) in (13), and calling $t=\delta_{k}^{2} \in[0,1]$, one obtains

$$
\langle\bar{u}, v\rangle \geq \frac{-\sqrt{t}+\sqrt{\mu} \sqrt{t \mu+(1-t) \mu_{1}}}{\sqrt{1+\mu} \sqrt{1+t \mu+(1-t) \mu_{1}}}=\left\langle\bar{u}, z_{t}\right\rangle,
$$

where $z_{t}$ is defined as in Step 1. Summarizing, we have shown the following extrapolation property: given an arbitrary point $v \in \Sigma_{n+1} \cap \mathcal{E}(A)$, there exists another point $z_{t}$ belonging to $\Sigma_{n+1} \cap \operatorname{bd}[\mathcal{E}(A)]$ and lying farther away from $\bar{u}$ than $v$.

Step 4: We prove that $(d) \Rightarrow(c)$. In view of the extrapolation property derived in Step 3, it suffices to prove that

$$
\left\langle\bar{u}, z_{t}\right\rangle \geq\langle\bar{u}, \bar{v}\rangle \quad \forall t \in[0,1],
$$

or equivalently, that $\phi:[0,1] \rightarrow \mathbb{R}$ attains its minimum at $t=1$. Under the assumption $\mu \leq 1+2 \mu_{1}$, one has of course $\mu_{1} \geq \max \{0,(\mu-1) / 2\}$. We consider two cases, according to the value of this maximum, i.e. to the sign of $\mu-1$. First we look at the right hand side of (11) as a function of $\mu_{1}$, which we will now denote as $\phi\left(t, \mu_{1}\right)$. By rewriting (11) as

$$
\phi\left(t, \mu_{1}\right)=\frac{-\sqrt{t}}{\sqrt{1+\mu} \sqrt{1+t \mu+(1-t) \mu_{1}}}+\frac{\sqrt{\mu}}{\sqrt{1+\mu} \sqrt{1+\frac{1}{t \mu+(1-t) \mu_{1}}}},
$$

one sees that $\phi(t, \cdot)$ is nondecreasing in the non-negative half-line for all $t \in$ $[0,1]$. Now we study the two cases of interest.

i) $\mu-1<0$. Observe that

$$
\begin{aligned}
\left\langle\bar{u}, z_{t}\right\rangle=\phi\left(t, \mu_{1}\right) \geq \phi(t, 0) & =\frac{-\sqrt{t}+\sqrt{\mu} \sqrt{\mu t}}{\sqrt{\mu+1} \sqrt{1+\mu t}} \\
& =\frac{\sqrt{t}(\mu-1)}{\sqrt{\mu+1} \sqrt{1+\mu t}} \\
& =\frac{\mu-1}{\sqrt{\mu+1} \sqrt{\frac{1}{t}+\mu}} .
\end{aligned}
$$


Since the rightmost expression in (15) is decreasing as a function of $t$ in the interval $[0,1]$, one gets

$$
\left\langle\bar{u}, \bar{z}_{t}\right\rangle \geq \phi(1,0)=(\mu-1) /(\mu+1)=\langle\bar{u}, \bar{v}\rangle
$$

for all $t \in[0,1]$, as we needed to prove.

ii) $\mu-1 \geq 0$. In this case we write

$$
\begin{aligned}
\left\langle\bar{u}, z_{t}\right\rangle & =\phi\left(t, \mu_{1}\right) \geq \phi\left(t, \frac{\mu-1}{2}\right) \\
& =\frac{-\sqrt{2 t}+\sqrt{\mu} \sqrt{t(\mu+1)+\mu-1}}{(\mu+1) \sqrt{t+1}} .
\end{aligned}
$$

For $t \in[0,1]$, denote by $\psi(t)$ as the rightmost expression of (16). The function $\psi:[0,1] \rightarrow \mathbb{R}$ is derivable and

$$
\begin{aligned}
(\mu+1)(t+1) \psi^{\prime}(t)= & \left(\frac{-\sqrt{2}}{2 \sqrt{t}}+\frac{\sqrt{\mu}(\mu+1)}{2 \sqrt{t(\mu+1)+\mu-1}}\right) \sqrt{t+1} \\
& -\left(\frac{-\sqrt{2 t}+\sqrt{\mu} \sqrt{t(\mu+1)+\mu-1}}{2 \sqrt{t+1}}\right) .
\end{aligned}
$$

After a tedious simplification one arrives at

$$
\psi^{\prime}(t)=\frac{1}{2(\mu+1)(t+1)^{3 / 2}}\left(-\sqrt{\frac{2}{t}}+\frac{2 \sqrt{\mu}}{\sqrt{t(\mu+1)+\mu-1}}\right) .
$$

It follows from (17) that $\psi^{\prime}(t) \leq 0$ if and only if $(\mu-1)(1-t) \geq 0$. We conclude that $\psi$ is nonincreasing in the whole interval $[0,1]$. Hence,

$$
\begin{aligned}
\left\langle\bar{u}, \bar{z}_{t}\right\rangle \geq \psi(t) \geq \psi(1) & =\frac{1}{\mu+1}\left(\frac{-\sqrt{2}+\sqrt{\mu} \sqrt{2 \mu}}{\sqrt{2}}\right) \\
& =\frac{\mu-1}{\mu+1}=\langle\bar{u}, \bar{v}\rangle
\end{aligned}
$$

for all $t \in[0,1]$. We reach again the desired inequality. 
Step 5: Completion of the proof. We have shown insofar that $(c) \Longleftrightarrow(d)$. By using a mutatis mutandis argument one proves similarly that $(b) \Longleftrightarrow(d)$. For completing the proof of the theorem it is now enough to observe that (a) is the conjunction of (b) and (c).

Corollary 1. For a proper critical pair $(\bar{u}, \bar{v})$ of an elliptic cone $K$ in $\mathbb{R}^{n+1}$ to be a Nash angular equilibrium, it is necessary and sufficient that

$$
\|\bar{u}-\bar{v}\| \geq \frac{\sqrt{2}}{2} \operatorname{diam}\left(K \cap \Sigma_{n+1}\right) .
$$

Proof. Let $K=\mathcal{E}(A)$ and suppose that the proper critical pair $(\bar{u}, \bar{v})$ is associated with the eigenvalue $\mu$. Since $\langle\bar{u}, \bar{v}\rangle=(\mu-1) /(\mu+1)$, one has $\|\bar{u}-\bar{v}\|=2 / \sqrt{\mu+1}$. On the other hand, the diameter of $K \cap \Sigma_{n+1}$ is attained with an antipodal pair of $K$, so one has

$$
\operatorname{diam}\left(K \cap \Sigma_{n+1}\right)=2 / \sqrt{\mu_{\min }(A)+1} .
$$

It is clear that the condition $\mu \leq 2 \mu_{\min }(A)+1$ is equivalent to

$$
\frac{2}{\sqrt{\mu+1}} \geq \frac{\sqrt{2}}{2} \frac{2}{\sqrt{\mu_{\min }(A)+1}},
$$

so the announced result follows from Theorem 3.

Corollary 2. For a proper critical angle $\theta$ of an elliptic cone $K$ to be a Nash angle, it is necessary and sufficient that

$$
\arccos \left[\frac{1+\cos \theta_{\max }(K)}{2}\right] \leq \theta .
$$

Proof. It is a matter of reformulating Corollary 1.

\subsection{Nash threshold coefficient of $\mathcal{E}(A)$}

It is reasonable to expect a Nash angular equilibrium to form an angle which is large, or at least not too small while compared with the maximal angle of the 
cone. This idea is corroborated by the relation (19) in Corollary 2 or, equivalently, by the relation (18) in Corollary 1 .

In connection with the above observation, recall that the Nash threshold coefficient of a nontrivial closed convex cone $K$ in $\mathbb{R}^{d}$ is the largest constant $\beta \in[0,1]$ such that

$$
\|\bar{u}-\bar{v}\| \geq \beta \operatorname{diam}\left(K \cap \Sigma_{d}\right) \quad \forall(\bar{u}, \bar{v}) \in \operatorname{Nash}(K),
$$

where $\operatorname{Nash}(K)$ stands for the set of all Nash angular equilibria of $K$. If $\beta_{K}$ denotes the Nash threshold coefficient of $K$, then the infimal-value

$$
\beta^{*}=\inf _{K \in \Xi\left(\mathbb{R}^{d}\right)} \beta_{K}
$$

corresponds to the largest constant $\beta$ for which the inequality (20) holds uniformly with respect to all elements in $\Xi\left(\mathbb{R}^{d}\right)$. After a considerable amount of effort we were able to prove in $[8$, Corollary 2$]$ that

$$
\left\{\begin{array}{l}
\text { in dimension } d \text { greater or equal than three, } \\
\text { the infimal-value } \beta^{*} \text { is equal to } \sqrt{3} / 3 .
\end{array}\right.
$$

Unless $K$ has a special structure, it is hopeless to derive a simple formula for evaluating $\beta_{K}$ itself. As far as elliptic cones are concerned, we are now in position to establish the following result.

Proposition 1. The Nash threshold coefficient of an elliptic cone $\mathcal{E}(A)$ is given by

$$
\beta_{\mathcal{E}(A)}=\sqrt{\frac{\mu_{\min }(A)+1}{\mu^{\star}(A)+1}},
$$

where $\mu^{\star}(A)$ denotes the largest eigenvalue of $A$ that is less or equal than $2 \mu_{\min }(A)+1$. In particular,

$$
\inf _{\substack{K \in \Xi\left(\mathbb{R}^{d}\right) \\ K \text { elliptic }}} \beta_{K}=\sqrt{2} / 2,
$$

and this infimum is attained by any elliptic cone $\mathcal{E}(A)$ such that $2 \mu_{\min }(A)+1$ is an eigenvalue of $A$. 
Proof. Let $\mu_{1} \leq \cdots \leq \mu_{n}$ be the eigenvalues of $A$. In view of Corollary 1 , the number $\beta_{\mathcal{E}(A)}$ corresponds to the largest constant $\beta \in[0,1]$ such that

$$
\frac{2}{\sqrt{\mu_{k}+1}} \geq \beta \frac{2}{\sqrt{\mu_{\min }(A)+1}}
$$

holds for any $k \in\{1, \ldots, n\}$ satisfying $\mu_{k} \leq 2 \mu_{\min }(A)+1$. A matter of simplification leads directly to the formula (21).

\section{Angular analysis of spectral cones}

We now work in a linear space of dimension $d=(1 / 2) n(n+1)$ with $n \geq 2$. More precisely, we are concerned with a special class of convex cones in

$$
\mathbb{S}_{n} \equiv \text { real symmetric matrices of size } n \times n .
$$

As usual, $\mathbb{S}_{n}$ is equipped with the Frobenius inner product $\langle A, B\rangle=\operatorname{trace}(A B)$ and the associated norm. For the sake of convenience we write $\Sigma\left(\mathbb{S}_{n}\right)=$ $\left\{A \in \mathbb{S}_{n}:\|A\|=1\right\}$ and reserve the symbol $\Sigma_{n}$ for the unit sphere in the Euclidean space $\mathbb{R}^{n}$.

For an arbitrary closed convex cone $\mathcal{M}$ in $\mathbb{S}_{n}$, the computation of the maximal angle $\theta_{\max }(\mathcal{M})$ is in general a cumbersome task. The same remark applies to the computation of the other possible critical angles. The purpose of this section is to derive useful calculus rules for computing critical angles at least for a special class of convex cones in $\mathbb{S}_{n}$.

Recall that a convex cone $\mathcal{M}$ in $\mathbb{S}_{n}$ is said to be spectral (or weakly unitarily invariant) if

$$
A \in \mathcal{M} \quad \Longrightarrow \quad U^{T} A U \in \mathcal{M} \text { for all } U \in \mathcal{O}_{n}
$$

with $\mathcal{O}_{n}$ denoting the group of orthonormal matrices of size $n \times n$. Notice, incidentally, that the concept of spectrality applies to an arbitrary set in $\mathbb{S}_{n}$ and not just for a convex cone.

The next two lemmas are part of the folklore on weakly unitarily invariant sets and functions, see for instance the references $[1,2,10,11,15]$. In the sequel the notation $\lambda(A)=\left(\lambda_{1}(A), \ldots, \lambda_{n}(A)\right)$ stands for the vector of eigenvalues of $A$ arranged in nondecreasing order, and $\operatorname{diag}(x)$ stands for the diagonal matrix whose entries on the diagonal are the components of the vector $x$. 
Lemma 2. A convex cone $\mathcal{M}$ in $\mathbb{S}_{n}$ is spectral if and only if there is a permutation invariant convex cone $K$ in $\mathbb{R}^{n}$ such that

$$
\mathcal{M}=\left\{A \in \mathbb{S}_{n}: \lambda(A) \in K\right\} .
$$

Furthermore, such $K$ is unique and given by

$$
K_{\mathcal{M}}=\left\{x \in \mathbb{R}^{n}: \operatorname{diag}(x) \in \mathcal{M}\right\} .
$$

One refers to $K_{\mathcal{M}}$ as the permutation invariant convex cone induced by $\mathcal{M}$. Recall that a set $K$ in $\mathbb{R}^{n}$ is called permutation invariant if $\Pi(K)=K$ for all $\Pi \in \Pi_{n}$, with $\Pi_{n}$ denoting the set of $n \times n$ permutation matrices.

\section{Lemma 3. One has:}

(a) the dual of a permutation invariant convex cone in $\mathbb{R}^{n}$ is a permutation invariant convex cone in $\mathbb{R}^{n}$.

(b) If $\mathcal{M}$ is a spectral convex cone in $\mathbb{S}_{n}$, then its dual $\mathcal{M}^{+}$is a spectral convex cone in $\mathbb{S}_{n}$. Furthermore, $\mathcal{M}^{+}$can be computed by using the formula

$$
\mathcal{M}^{+}=\left\{A \in \mathbb{S}_{n}: \lambda(A) \in K_{\mathcal{X}}^{+}\right\} \text {. }
$$

The most popular example of spectral convex cone in $\mathbb{S}_{n}$ is the Loewner cone of positive semidefinite symmetric matrices. A list of more elaborate spectral convex cones includes

$$
\begin{array}{ll}
\mathcal{M}=\left\{A \in \mathbb{S}_{n}: \lambda_{1}(A)+\ldots+\lambda_{m}(A) \geq 0\right\} & (1 \leq m \leq n-1), \\
\mathcal{M}=\left\{A \in \mathbb{S}_{n}: \gamma \sum_{i=r}^{n} \lambda_{i}(A) \leq \sum_{i=1}^{m} \lambda_{i}(A)\right\} & (1 \leq m, r \leq n, \gamma \geq 0), \\
\mathcal{M}=\left\{A \in \mathbb{S}_{n}: \max \left\{0, \lambda_{n}(A)\right\}+\gamma\|A\| \leq \delta \operatorname{trace}(A)\right\} & (\gamma \geq 0, \delta \in \mathbb{R}), \\
\mathcal{M}=\left\{A \in \mathbb{S}_{n}: \sqrt{\left[\lambda_{n}(A)-\lambda_{1}(A)\right]^{2}+\|A\|^{2}} \leq \delta \operatorname{trace}(A)\right\} & (\delta \geq 0), \\
\mathcal{M}=\left\{A \in \mathbb{S}_{n}: \gamma\|A\| \leq \operatorname{trace}(A)\right\} & (\gamma>0),
\end{array}
$$

just to mention a few examples. In all cases it is easy to recognize which is the corresponding permutation invariant convex cone $K_{\mathcal{M}}$. If $x^{\uparrow}$ denotes the 
vector which is obtained by rearranging in nondecreasing order the components of $x \in \mathbb{R}^{n}$, then one gets

$$
\begin{aligned}
& K_{\mathcal{M}}=\left\{x \in \mathbb{R}^{n}: x_{1}^{\uparrow}+\ldots+x_{m}^{\uparrow} \geq 0\right\}, \\
& K_{\mathcal{M}}=\left\{x \in \mathbb{R}^{n}: \gamma \sum_{i=\ell}^{n} x_{i}^{\uparrow} \leq \sum_{i=1}^{m} x_{i}^{\uparrow}\right\},
\end{aligned}
$$

and so on. The example (23) is perhaps the most interesting one since it appears in concrete problems of optimization [12] and principal components analysis [14].

Be aware that $K_{\mathcal{M}}$ may posses some properties that are lacking in $\mathcal{M}$, think for instance of polyhedrality. It is not difficult to see that the convex cone (24) is polyhedral, but the spectral convex cone (23) is not. The lack of polyhedrality in (23) is due to a "curvature" effect introduced by the eigenvalue functions $\lambda_{i}: \mathbb{S}_{n} \rightarrow \mathbb{R}$.

\subsection{Antipodal pairs in a spectral cone}

Is there any link between the angular structure of $\mathcal{M}$ and that of $K_{\mathcal{M}}$ ? Answering this question is not a trivial matter. Our first task will be comparing the maximal angle

$$
\theta_{\max }(\mathcal{M})=\sup _{\substack{A, B \in \mathcal{M} \\\|A\|=1,\|B\|=1}} \arccos \langle A, B\rangle
$$

of the spectral cone $\mathcal{M}$ in $\mathbb{S}_{n}$ and the maximal angle

$$
\theta_{\max }\left(K_{\mathcal{M}}\right)=\sup _{\substack{u, v \in K_{\mathcal{X}} \\\|u\|=1,\|v\|=1}} \arccos \langle u, v\rangle .
$$

of the permutation invariant cone $K_{\mathcal{M}}$. The last term is easier to evaluate because $K_{\mathcal{M}}$ has in principle a simpler structure and, in any case, it lives in a vector space of lower dimension.

As explained in the next theorem, the antipodal pairs of $\mathcal{M}$ and those of $K_{\mathcal{M}}$ are related through the eigenvalue map $\lambda: \mathbb{S}_{n} \rightarrow \mathbb{R}^{n}$. We start by establishing a commutation principle for optimization problems with spectral data. Recall that a spectral set in $\mathbb{S}_{n}$ is defined by means of the relation (22). Similarly, a function $\Phi$ on $\mathbb{S}_{n}$ is said to be spectral (or weakly unitarily invariant) if

$$
\Phi\left(U^{T} A U\right)=\Phi(A) \text { for all } U \in \mathcal{O}_{n} .
$$


Lemma 4 (Commutation principle). Let $\mathcal{N} \subset \mathbb{S}_{n}$ be a spectral set and $\Phi$ : $\mathbb{S}_{n} \rightarrow \mathbb{R}$ be a spectral function. Let $\bar{A}, \bar{B} \in \mathbb{S}_{n}$. If $\bar{B}$ is a local minimum (or a local maximum) over $\mathcal{N}$ of the fonction $\langle\bar{A}, \cdot\rangle+\Phi(\cdot)$, then $\bar{A}$ and $\bar{B}$ commute.

Proof. Suppose that $\bar{B}$ is a local minimum of $\langle\bar{A}, \cdot\rangle+\Phi(\cdot)$ over $\mathcal{N}$. This means that $\bar{B} \in \mathcal{N}$ and

$$
\langle\bar{A}, \bar{B}\rangle+\Phi(\bar{B}) \leq\langle\bar{A}, B\rangle+\Phi(B) \quad \forall B \in \mathcal{N} \cap \mathbb{O}_{\varepsilon}(\bar{B})
$$

with $\mathbb{O}_{\varepsilon}(\bar{B})$ denoting an open ball of radius $\varepsilon>0$ and center $\bar{B}$. Take $\bar{X} \in \mathcal{O}_{n}$ so that

$$
\bar{X}^{T} \bar{B} \bar{X}=E=\operatorname{diag}(\lambda(\bar{B})) .
$$

By definition of spectral set, $X E X^{T}$ belong to $\mathcal{N}$ for all $X \in \mathcal{O}_{n}$. By a continuity argument, there is a small $\delta>0$ such that $X E X^{T} \in \mathbb{O}_{\varepsilon}(\bar{B})$ whenever $X \in$ $\mathbb{O}_{\delta}(\bar{X})$. In view of $(25)$, one gets

$$
\left\langle\bar{A}, \bar{X} E \bar{X}^{T}\right\rangle+\Phi\left(\bar{X} E \bar{X}^{T}\right) \leq\left\langle\bar{A}, X E X^{T}\right\rangle+\Phi\left(X E X^{T}\right) \quad \forall X \in \mathcal{O}_{n} \cap \mathbb{O}_{\delta}(\bar{X}) .
$$

But, by spectrality of $\Phi$, the terms $\Phi\left(\bar{X} E \bar{X}^{T}\right)$ and $\Phi\left(X E X^{T}\right)$ cancel out. The conclusion is that $\bar{X}$ is a local solution to the optimization problem

$$
\begin{aligned}
& \text { minimize } f(X)=\left\langle\bar{A}, X E X^{T}\right\rangle \\
& \text { with respect to } X \in \mathcal{O}_{n},
\end{aligned}
$$

and hence it satisfies the first order necessary optimality conditions for this problem. Before writing down these optimality conditions, observe that $f$ is not defined on $\mathbb{S}_{n}$ but on the space $\mathbb{M}_{n}$ of arbitrary real matrices of size $n \times n$. The Frobenius inner product on $\mathbb{M}_{n}$ is given by $\langle X, Y\rangle=\operatorname{trace}\left(X^{T} Y\right)$. By rewriting the contraint (26) as $X X^{T}=I$ and introducing the Lagrangean function

$$
(X, M) \in \mathbb{M}_{n} \times \mathbb{M}_{n} \mapsto L(X, M)=\left\langle\bar{A}, X E X^{T}\right\rangle-\left\langle M, X X^{T}-I\right\rangle,
$$

we see that $\bar{X}$ satisfies

$$
\begin{aligned}
& \nabla_{M} L(\bar{X}, \bar{M})=\bar{X} \bar{X}^{T}-I=0, \\
& \nabla_{X} L(\bar{X}, \bar{M})=2 \bar{A} \bar{X} E-\left(\bar{M}+\bar{M}^{T}\right) \bar{X}=0,
\end{aligned}
$$


for some $\bar{M} \in \mathbb{M}_{n}$. Since $\bar{X}^{-1}=\bar{X}^{T}$ by (27), we get from (28) that

$$
\bar{A} \bar{B}=\bar{A}\left(\bar{X} E \bar{X}^{T}\right)=(1 / 2)\left(\bar{M}+\bar{M}^{T}\right)
$$

is a symmetric matrix. It follows that $\bar{A} \bar{B}=(\bar{A} \bar{B})^{T}=\bar{B} \bar{A}$. The case of a local maximum is treated in a similar way.

It is possible to derive more sophisticated versions of the above commutation principle but Lemma 4 is general enough to cover all our needs. Everything is now ready to state:

Theorem 4. A spectral closed convex cone $\mathcal{M}$ in $\mathbb{S}_{n}$ and the associated permutation invariant closed convex cone $K_{\mathcal{M}}$ have the same maximal angle, i.e., $\theta_{\max }(\mathcal{M})=\theta_{\max }\left(K_{\mathcal{M}}\right)$. Furthermore, the following statements are equivalent:

(a) $(\bar{A}, \bar{B}) \in \mathbb{S}_{n} \times \mathbb{S}_{n}$ is an antipodal pair of $\mathcal{M}$.

(b) there exist an antipodal pair $(\bar{u}, \bar{v})$ of $K_{\mathcal{M}}$ and a matrix $Q \in \mathcal{O}_{n}$ such that $\bar{A}=Q \operatorname{diag}(\bar{u}) Q^{T}$ and $\bar{B}=Q \operatorname{diag}(\bar{v}) Q^{T}$.

Proof. Let $(\bar{u}, \bar{v})$ be an antipodal pair of $K_{\mathcal{M}}$. Write $\bar{A}=\operatorname{diag}(\bar{u})$ and $\bar{B}=$ $\operatorname{diag}(\bar{v})$. One has $\|\bar{A}\|=\|\bar{u}\|=1,\|\bar{B}\|=\|\bar{v}\|=1$, and also $\lambda(\bar{A})=\bar{u}^{\uparrow}$, $\lambda(\bar{B})=\bar{v}^{\uparrow}$. Since $K_{\mathcal{M}}$ is permutation invariant, the vectors $\bar{u}^{\uparrow}$ and $\bar{v}^{\uparrow}$ remain in $K_{\mathcal{M}}$, and therefore $\bar{A}, \bar{B} \in \mathcal{M}$. The conclusion is that

$$
\theta_{\max }(\mathcal{M}) \geq \arccos \langle\bar{A}, \bar{B}\rangle=\arccos \langle\bar{u}, \bar{v}\rangle=\theta_{\max }\left(K_{\mathcal{M}}\right) .
$$

The reverse inequality is obtained by exploiting the commutation principle stated in Lemma 4. The proof runs as follows. Take matrices $\bar{A}, \bar{B} \in \mathcal{M}$ of unit length realizing the maximal angle in $\mathcal{M}$, i.e.,

$$
\langle\bar{A}, \bar{B}\rangle=\min _{A, B \in \mathcal{M} \cap \Sigma\left(\mathbb{S}_{n}\right)}\langle A, B\rangle .
$$

In particular, $\bar{B}$ is a minimizer of the linear form $\langle\bar{A}, \cdot\rangle$ over the spectral set $\mathcal{M} \cap \Sigma\left(\mathbb{S}_{n}\right)$. Lemma 4 implies that $\bar{A}$ and $\bar{B}$ commute. Hence, $\bar{A}$ and $\bar{B}$ can be simultaneously diagonalized by means of a matrix $Q \in \mathcal{O}_{n}$. This means that

$$
Q^{T} \bar{A} Q=\operatorname{diag}(\bar{u}) \quad \text { and } \quad Q^{T} \bar{B} Q=\operatorname{diag}(\bar{v})
$$


for suitable vectors $\bar{u}, \bar{v} \in \mathbb{R}^{n}$. Hence,

$$
\langle\bar{A}, \bar{B}\rangle=\left\langle Q \operatorname{diag}(\bar{u}) Q^{T}, Q \operatorname{diag}(\bar{v}) Q^{T}\right\rangle=\langle\operatorname{diag}(\bar{u}), \operatorname{diag}(\bar{v})\rangle=\langle\bar{u}, \bar{v}\rangle .
$$

Observe also that $\bar{u}, \bar{v}$ are unit vectors and, by spectrality of $\mathcal{M}$, they are in $K_{\mathcal{M}}$. Hence, one gets

$$
\langle\bar{A}, \bar{B}\rangle \geq \inf _{u, v \in K_{\mathcal{M}} \cap \Sigma_{n}}\langle u, v\rangle
$$

and the desired inequality $\theta_{\max }(\mathcal{M}) \leq \theta_{\max }\left(K_{\mathcal{N}}\right)$. The second part of the theorem is implicit in the above proof.

\subsection{Critical pairs in a spectral cone}

We now compare the angular spectra of $\mathcal{M}$ and $K_{\mathcal{M}}$. The commutation principle stated in Lemma 4 plays again a crucial role.

Theorem 5. A spectral closed convex cone $\mathcal{M}$ in $\mathbb{S}_{n}$ and the associated permutation invariant closed convex cone $K_{\mathcal{M}}$ have the same collection of proper critical angles, i.e., $\Omega(\mathcal{M})=\Omega\left(\mathcal{K}_{\mathcal{M}}\right)$. Furthermore, the following statements are equivalent:

(a) $(\bar{A}, \bar{B}) \in \mathbb{S}_{n} \times \mathbb{S}_{n}$ is a critical pair of $\mathcal{M}$.

(b) there exist a critical pair $(\bar{u}, \bar{v})$ of $K_{\mathcal{M}}$ and a matrix $Q \in \mathcal{O}_{n}$ such that $\bar{A}=Q \operatorname{diag}(\bar{u}) Q^{T}$ and $\bar{B}=Q \operatorname{diag}(\bar{v}) Q^{T}$.

Proof. Suppose that $\bar{A}, \bar{B} \in \mathcal{M} \cap \Sigma\left(\mathbb{S}_{n}\right)$ satisfy the criticality conditions

$$
\begin{gathered}
\bar{B}-\langle\bar{A}, \bar{B}\rangle \bar{A} \in \mathcal{M}^{+}, \\
\bar{A}-\langle\bar{A}, \bar{B}\rangle \bar{B} \in \mathcal{M}^{+} .
\end{gathered}
$$

We shall prove that $\bar{A}$ and $\bar{B}$ commute. To do this, we come back to the very definition of a critical pair. It is not difficult to see that the system (30)-(31) can be written in the form

$$
\begin{aligned}
& -[\bar{B}-\eta \bar{A}] \in \partial \Psi_{\mathcal{M}}(\bar{A}), \\
& -[\bar{A}-\eta \bar{B}] \in \partial \Psi_{\mathcal{M}}(\bar{B}),
\end{aligned}
$$


with $\partial$ standing for the subdifferential operator in the sense of convex analysis, $\Psi_{\mathcal{M}}$ denoting the indicator function of $\mathcal{M}$, and $\eta=\langle\bar{A}, \bar{B}\rangle$. Let us examine more closely for instance the relation (32). Standard rules of convex analysis show that (32) amounts to saying that $\bar{B}$ minimizes the linear function $\langle\bar{A}-\eta \bar{B}, \cdot\rangle$ over the set $\mathcal{M}$. In view of Lemma 4 , one obtains the commutation property

$$
(\bar{A}-\eta \bar{B}) \bar{B}=\bar{B}(\bar{A}-\eta \bar{B}),
$$

confirming in this way that $\bar{A} \bar{B}=\bar{B} \bar{A}$. By proceeding to a simultaneous diagonalization as in (29) one obtains

$$
\begin{aligned}
& Q \operatorname{diag}(\bar{v}) Q^{T}-\eta Q \operatorname{diag}(\bar{u}) Q^{T} \in \mathcal{M}^{+}, \\
& Q \operatorname{diag}(\bar{u}) Q^{T}-\eta Q \operatorname{diag}(\bar{v}) Q^{T} \in \mathcal{M}^{+} .
\end{aligned}
$$

By spectrality of $\mathcal{M}^{+}$we can drop the common orthonormal transformation $Q$ and write simply

$$
\begin{aligned}
& \operatorname{diag}(\bar{v}-\eta \bar{u}) \in \mathcal{M}^{+}, \\
& \operatorname{diag}(\bar{u}-\eta \bar{v}) \in \mathcal{M}^{+} .
\end{aligned}
$$

By recalling Lemmas 2 and 3, one sees that $(\bar{u}, \bar{v})$ is necessarily a critical pair of $K_{\mathcal{M}}$. The proof of the reverse implication $(b) \Rightarrow(a)$ is omitted since it offers no difficulty.

Remark 2. As one sees from Theorem 5, a critical pair $(\bar{A}, \bar{B})$ of $\mathcal{M}$ is formed necessarily with a couple of commuting matrices. The components of the vectors $\bar{u}, \bar{v}$ produced by the simultaneous diagonalization (29) may not be arranged in a similar order, i.e., there may be no common permutation matrix $\Pi \in \Pi_{n}$ such that $\Pi u=u^{\uparrow}$ and $\Pi v=v^{\uparrow}$. This explains why we cannot infer that $(\lambda(\bar{A}), \lambda(\bar{B}))$ is a critical pair of $K_{\mathcal{M}}$.

Remark 3. Recall that the critical angles of a closed convex cone can be separated into two disjoint groups: Nash angles and ordinary critical angles. Suppose that $(\bar{A}, \bar{B})$ is a Nash angular equilibrium of $\mathcal{M}$, i.e., one has the combination of the following two conditions:

$$
\begin{gathered}
\bar{B} \text { minimizes }\langle\bar{A}, \cdot\rangle \text { over } \mathcal{M} \cap \Sigma\left(\mathbb{S}_{n}\right), \\
\bar{A} \text { minimizes }\langle\cdot, \bar{B}\rangle \text { over } \mathcal{M} \cap \Sigma\left(\mathbb{S}_{n}\right) .
\end{gathered}
$$


In view of Lemma 4 , either one of these conditions imply that $\bar{A} \bar{B}=\bar{B} \bar{A}$. By plugging $\bar{A}=Q \operatorname{diag}(\bar{u}) Q^{T}$ and $\bar{B}=Q \operatorname{diag}(\bar{v}) Q^{T}$ into (33)-(34) and working out the details, one concludes that $(\bar{u}, \bar{v})$ is necessarily a Nash angular equilibrium of $K_{\mathcal{M}}$.

\section{REFERENCES}

[1] B. Dacorogna and P. Maréchal, Convex $S O(N) \times S O(n)$-invariant functions and refinements of von Neumann's inequality. Ann. Fac. Sci. Toulouse Math., 16 (2007), 71-89.

[2] C. Davis, All convex invariant functions of hermitian matrices. Archiv der Math., 8 (1957), 276-278.

[3] Z.Q. Feng, M. Hjiaj, G. de Saxcé and Z. Mróz, Effect of frictional anisotropy on the quasistatic motion of a deformable solid sliding on a planar surface. Comput. Mech., 37 (2006), 349-361.

[4] A. Iusem and A. Seeger, Measuring the degree of pointedness of a closed convex cone: a metric approach. Math. Nachrichten, 279 (2006), 599-618.

[5] A. Iusem and A. Seeger, On vectors achieving the maximal angle of a convex cone. Math. Programming, 104 (2005), 501-523.

[6] A. Iusem and A. Seeger, On convex cones with infinitely many critical angles. Optimization, 56 (2007), 115-128.

[7] A. Iusem and A. Seeger, Searching for critical angles in a convex cone. Math. Programming, 2007 , in press.

[8] A. Iusem and A. Seeger, Antipodal pairs, critical pairs, and Nash angular equilibria in convex cones. Optimization Methods and Software, submitted.

[9] A. Iusem and A. Seeger, Antipodality in convex cones and distance to unpointedness, May 2006, submitted.

[10] A. Lewis, Convex analysis of on the Hermitian matrices. SIAM J. Optim., 6 (1996), 164-177.

[11] J.E. Martinez-Legaz, On convex and quasiconvex spectral functions. Proceedings of the 2nd Catalan Days on Applied Mathematics (Odeillo, 1995), 199-208, Collect. Études, Presses Univ. Perpignan, Perpignan, 1995.

[12] M. Overton and R.S. Womersley, Optimality conditions and duality theory for minimizing sums of the largest eigenvalues of symmetric matrices. Math. Programming, 62 (1993) 321-357.

[13] J. Peña and J. Renegar, Computing approximate solutions for conic systems of constraints. Math. Programming, 87 (2000), 351-383.

[14] G. Saporta, Probabilités, Analyse des Donnés et Statistique, Editions Technip, Paris, 1990.

[15] A. Seeger, Convex analysis of spectrally defined matrix functions. SIAM J. Optim., 7 (1997), 679-696. 
[16] D.S. Wang and L.N. Medgyesi-Mitschang, Electromagnetic scattering from finite circular and elliptic cones. IEEE Trans. Antennas and Propagation, 33 (1985), 488-497.

[17] H.W. Zhang, S.Y. He, X.S. Li, and P. Wriggers, A new algorithm for numerical solution of 3D elastoplastic contact problems with orthotropic friction law. Comput. Mech., 34 (2004), $1-14$. 